\title{
Collationnement des informations concernant l'effet d'échelle sur les rendements et les débits dans les turbines Pelton
}

\author{
PAR M. VERCASSON, \\ GENTRE DE RECHERCHES ET D'ESSAIS DE CHATOI: (E.D.F.)
}

\begin{abstract}
Ce texte a été mis au point dans le cadre de la section machines hydrauliques de la Sociélé Hydrotechnique de France, par le groupe de travail no 4 dont la composition était la suivante: MM. Vercasson (G.R.E.C.-E.D.F.), Combette (SO.GR.E.A.H.), Fauconnet (Ateliers des Charmilles), OnTunis (Aleliers de constructions mécaniques de Vevey, Winta (E.D.F. Division Technique Générale).

L'effet d'échelle résultant de la variation de la chute seule ou de la variation simmltanée de la chute et des dimensions est mis en évidence par l'exposé d'un certain nombre de résultals numériques oblenus dans différents laboratoires. Quelques interprétations de ces résultats sont également proposées.
\end{abstract}

\section{INTRODUCTION}

La Section «Machines hydrauliques» de la Société Hydrotechnique de France nous a demandé de collationner les informations concernant l'effet d'échelle sur les rendements et les débits dans les turbines Pelton.

Ce type de turbine n'a pas fait, dans ce domaine, l'objet de recherches aussi nombreuses que les turbines à réaction et, en tout cas, ces recherches n'ont abouti, à notre connaissance, à aucune formule de transposition pratiquement utilisée.

Dans ces conditions, nous pouvons nous demander s'il existe réellement un problème, d'autant plus que le code d'essai de la C.E.I. relatif aux essais de réception sur modèle réduit mentionne au paragraphe 3.2.2.2. :
«Pour les turbines Pelton, pas de formule d'effet d'échelle; rendement prototype $=$ rendement modèle $\gg$.

En fait, nous pensons, à la lecture des documents réunis, que cette position est due à la discordance des effets d'échelle constatés par les différents expérimentateurs et cela justifie l'étude entreprise.

Nous remercions ici les personnes et les organismes qui ont aimablement répondu à notre questionnaire :

MM.

Borciani : Société Franco-Tosi, Milan; Dziallas : J. M. Voith G.m.b.H., Heidenheim; Gerber : Ecole Polytechnique fédérale, Zurich; Huguenin: Ecole nationale des Arts el Métiers, Paris; 
Novgaro : E.N.S.E.E.H.T., Toulouse;

OsterWaLder : Etablissements Escher Wyss, Zurich;

SANTON : E.N.S.E.H.G., Grenoble;

Trnot : Ecole nationale des Arts et Métiers, Paris;

VIANO : Etablissements Riva, Milan;

SPEnCER : National Engineering laboratory, Glasgow;

ZIEGLER : Anstalt für Strömungsmaschinen, Autriche;

Etablissements Bell S.A., Lucerne;

Intitut national des Sciences Appliquées, Lyon;

Pelton Dryision of Baldwin Lima Hamilton Corporation, San Francisco;

Bureau of Reclamation, Denver;

Et tout particulièrement :

MM.

Combette : SO.GR.E.A.H., Grenoble;

Fauconnet : Ateliers des Charmilles, Genève;

OrtuiEB : Ateliers de Constructions mécaniques de Vevey;

Willm : Electricité de France, Grenoble;

membres du groupe de travail qui ont pris une part active à la rédaction de ce texte.

Afin de faciliter la rédaction de ce rapport, nous avons adressé aux organismes consultés un questionnaire dont le plan sera intégralement repris ici.

I - Renseignements bibliographiques;

II - Constatation de l'effet d'échelle;

III - Interprétation des résultats. Séparation des pertes;

IV - Autres études théoriques et expérimentales sur ce sujet.

\section{Présentation des résultats}

Le mode de présentation des résultats varie considérablement d'un expérimentateur à l'autre et nous avons été obligés de renoncer au choix d'un système unique et surtout au travail matériel considérable que représente la transposition des résultats d'un système dans l'autre, lorsqu'elle est possible. Néanmoins les notations utilisées sont certainement familières à tous les lecteurs.

\section{I. - RENSEIGNEMENTS BIBLIOGRAPHIQUES}

$1 \mathrm{~J}$. Dodu, - Similitude des jets liquides à grande vitesse. Symposium A.I.R.H., Nice (1960).
2. M. Gariel et J. Duport. - Modèles réduits de machines hydrauliques. Convegno di Venezia (octobre 1955).

3. S. Ferry. - Examen comparatif des pertes dans diverses turbines Pelton. Symposium A.I.R.H., Nice (1960).

4. H. Gerber. - Ventilationsverluste von Freistrahlturbinen Laufrädern. Bulletin de 'Association Suisse des Electriciens, $\mathrm{n}^{\circ} \mathrm{g}$ (1956).

5. H. Gerber. - Wassermessung in Freistrahlturbinenanlagen. Schweizerische Bauzeitung, $\mathrm{n}^{\circ} 14$ (1941).

6. H. Gerber. - La turbine Sfindex à courant annulaire. La Houille Blanche, $\mathrm{n}^{\circ} 6$ (décembre 1955).

7. R. Lowy. - Efficiency analysis of Pelton Wheels. Transactions of A.S.M.E., Vol. 6 (août 1944).

8. R. Vercellini. - Contribution à l'analyse des résultats d'essais de turbines Pelton. Informations techniques Charmilles, $\mathrm{n}^{\circ} 8$ (1961).

9. G. Willm. - Observations sur les Pelton. V's Journées de l'Hydraulique, Aix (1958)

10. Divers auteurs. - Symposium on laboratory testing of hydraulic turbine models in relation to field performance. Transactions of A.S.M.E., vol. 80 (octobre 1958).

\section{II. - EXPOSE DES RESULTATS D'ESSAIS METTANT EN EVIDENCE UN EFFET D'ÉCHELLE SOIT SUR LE DEBIT SOIT SUR LE RENDEMENT}

Nous avons arbitrairement divisé ce chapitre en trois :

A - Influence de la chute seule;

$B$ - Influence de la chute et des dimensions;

C - Influence des dimensions seules.

\section{A - Essai d'une turbine sous différentes chutes.}

Ce type d'essai est évidemment le plus facile à réaliser avec précision, tout au moins sur modèle réduit. Le plus souvent, un même modèle est capable de fonctionner sous différentes chutes et parfois les mêmes appareils de mesure peuvent être employés.

Il ne se pose donc aucun problème de similitude géométrique et il suffit de réaliser des me- 
sures sensibles et fidèles pour pouvoir faire des comparaisons valables.

Dans l'ensemble des laboratoires consultés, les modèles essayés peuvent être à axe horizontal et vertical et il est possible d'installer 1 ou 2 jets sur les modèles à axe horizontal et 2,4 ou 6 jets sur les modèles à axe vertical. L'essai peut avoir lieu avec ou sans bâti-capote.

La détermination de la chute nette se fait entre l'entrée de la turbine, prise en général à l'entrée de l'injecteur — ou du collecteur dans le cas de turbine à jets multiples - et la sortie, prise à la cote du point de contact du cercle centré sur l'axe de rotation et tangent à l'axe du jet. Dans le cas de jets multiples, la cote de la sortie est prise égale à la cote moyenne des points définis comme ci-dessus. L'énergie cinétique de l'eau à la sortie de la roue est évidemment comptée parmi les pertes.

La puissance mesurée est la puissance totale fournie par la roue, c'est-à-dire qu'elle comprend la puissance dissipée dans les paliers de la turbine et du frein servant à la mesure du couple.

Les chutes d'essais utilisées, qui étaient autrefois comprises entre 25 et $100 \mathrm{~m}$, tendent à augmenter et l'on note des chutes d'essais pouvant atteindre $600 \mathrm{~m}$.

A ce sujet rappelons les prescriptions du code d'essai de la C.E.I. en ce qui concerne les turbines Pelton:

- largeur d'auget : $l>80 \mathrm{~mm}$;

-. chute d'essai : $\mathrm{H}>40 \mathrm{~m}$;

- Nombre de Reynolds $=\frac{l \sqrt{2 g \mathrm{H}}}{\nu}>3,5.10^{6}$.

Tous les résultats recueillis concernent des essais sur modèle réduit.

1. Document Bell S.A.

Aucun effet d'échelle sur les rendements n'est constaté entre les essais sous 25 et $50 \mathrm{~m}$ de chute.

$2^{\circ}$ Documents Cernon (E.D.F. - SO.GR.E.A.H.).

Les résultats relatés par la figure $n^{\circ} 1$ font partie d'un ensemble de 22 essais comparatifs entre 11 modèles différents sous 60 et $600 \mathrm{~m}$ de chute.

Les notations utilisées sont les suivantes :

$$
\begin{aligned}
\mathrm{H} & =\text { chute nette; } \\
\mathrm{N} & =\text { puissance; } \\
n & =\text { vitesse de rotation; } \\
n_{e 1} & =\frac{n}{\sqrt{\mathrm{H}}} \\
\mathrm{N}_{e 1} & =\frac{\mathrm{N}}{\mathrm{H} \sqrt{\mathrm{H}}}
\end{aligned}
$$

Nous pouvons constater sur ce graphique la bonne coïncidence des résultats obtenus sous $600 \mathrm{~m}$ par la méthode thermodynamique et par les mesures au frein et au déversoir.

Les deux essais sous $60 \mathrm{~m}$ montrent que, sous cette chute, l'influence du bâti-capote est négligeable.

Pour les 11 essais comparatifs, les résultats sont qualitativement semblables : en passant de 60 à $600 \mathrm{~m}$, nous constatons :

- un abaissement général des rendements;

- un déplacement du point de rendement optimal vers les faibles vitesses $\left(n_{e, 1}\right)$ et vers les faibles puissances $\left(\mathrm{N}_{e 1}\right)$.

Ces essais n'ont pas permis de mettre en évidence un effet d'échelle notable sur les débits.

$3^{\circ}$ Documents Charmilles (tirées de Informations Techniques Charmilles, réf. $\mathrm{n}^{\circ} 8$ ).

Ces résultats concernent un modèle de turbine à axe vertical, à quatre jets de $0,539 \mathrm{~m}$ de diamètre Pelton.

Le mode de représentation sur la figure 2 est celui préconisé par S. Ferry [réf. n*3].

La puissance hydraulique et les pertes sont exprimées en \% de la puissance hydraulique de référence définie par :

$$
\mathrm{N}_{\text {réf. }}=Z_{0} \rho \frac{\pi}{4}\left(\frac{d_{i}}{\mathrm{~B}_{2}}\right)^{2} \quad \mathrm{~B}_{2}{ }^{2} \sqrt{2 g \mathrm{H}_{n}} g \mathrm{H}_{n}
$$

en choisissant $\left(d_{j} / \mathrm{B}_{2}\right)=0,34236$.

où :

$$
\begin{aligned}
\mathrm{Z}_{0} & =\text { nombre de jets; } \\
\mathrm{p} & =\text { masse volumique de l'eau; } \\
d_{j} & =\text { diamètre du jet; } \\
\mathrm{B}_{2} & =\text { largeur intérieure de l'auget; } \\
\mathrm{H}_{n} & =\text { chute nette. }
\end{aligned}
$$

\begin{tabular}{|c|c|}
\hline Chute d'essai..... & 76,2 \\
\hline Diamètre Pelton...... & 488,95 \\
\hline Diamètre de l'injecteur ( $\mathrm{mm}$ ) & 152,4 \\
\hline$t \ldots \ldots(\mathrm{mm})$ & 43,18 \\
\hline Meilleur & 89,8 \\
\hline
\end{tabular}

$4^{\circ}$ Documents Pelton (Division of Baldwin Lima Hamilton Corporation).

\section{Modèle à axe horizontal}

\begin{tabular}{l|c|c|r} 
Chute d'essai ....... & $579,12 \mathrm{~m}$ & $304,8 \mathrm{~m}$ & $152,4 \mathrm{~m}$ \\
Diamètre Pelton... & $336,5 \mathrm{~mm}$ & & \\
Largeur de capote. & $228,6 \mathrm{~mm}$ & & \\
Meilleur rendement. & $79,6 \%$ & $79,2 \%$ & $78,2 \%$
\end{tabular}

\section{Modèle $\dot{a}$ axe vertical à six jets}

\section{$5^{\circ}$ Documents SO.GR.E.A.H.}

Pratiquement aucun effet d'échelle n'est constaté sur le rendement entre les essais sous 60 et $120 \mathrm{~m}$. 


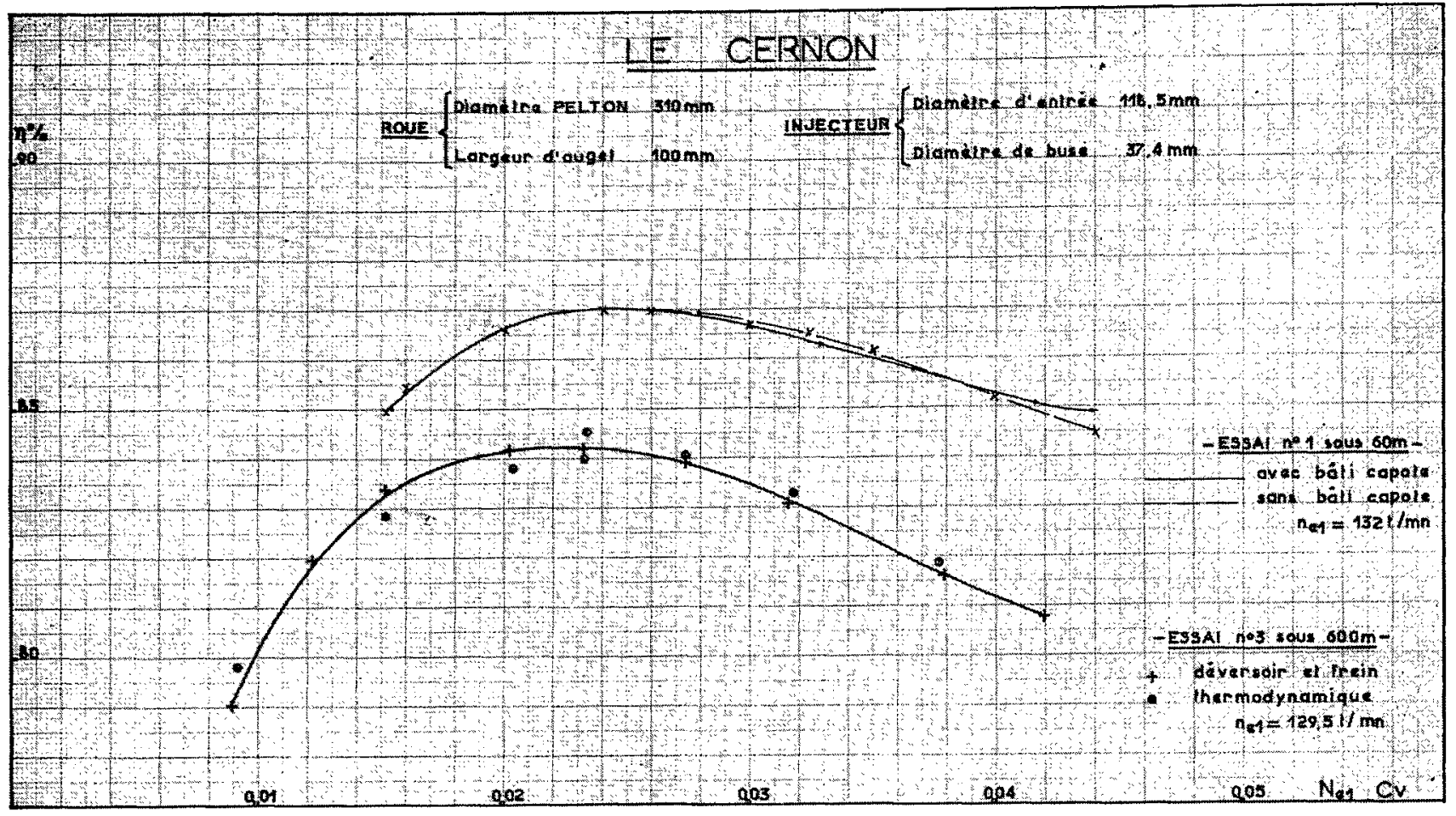

Fin. 1.

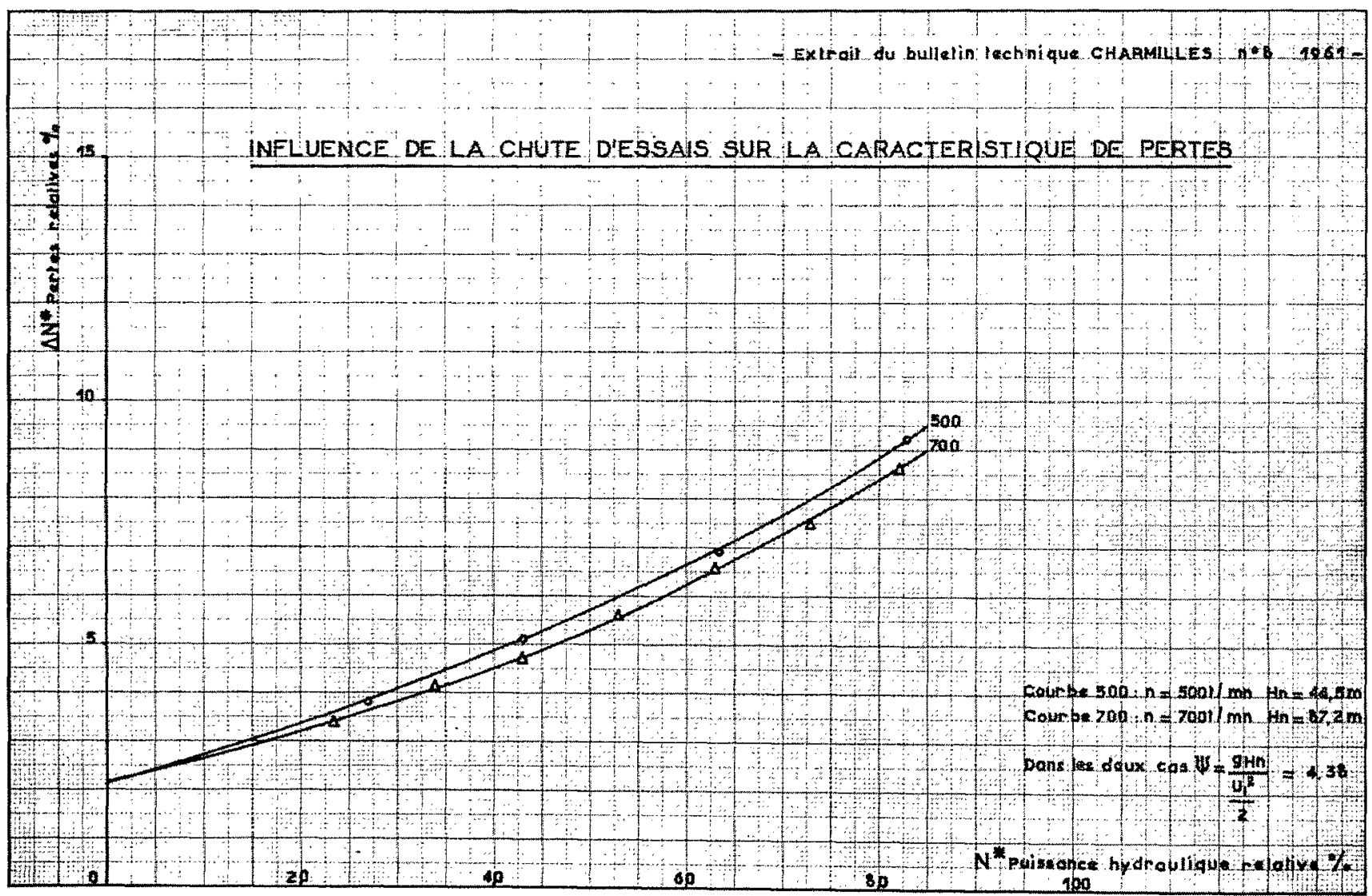

Fig. 2. 
$6^{\circ}$ Documents Vevey (fig. 3 et 4 ).

Le modèle utilisé présente les caractéristiques suivantes :

- diamètre Pelton $453 \mathrm{~m}$;

-- diamètre extérieur $586,3 \mathrm{~mm}$;

-- largeur intérieure d'auget 128,6 mm;

-. diamètre maximal du jet $42,5 \mathrm{~mm}$.

Les essais ont été réalisés avec le bâti-capote normal (fig. 4), avec le bâti-capote étroit (fig. 3) et sans le bâti-capote.

Le rendement de la turbine est représenté en fonction du débit sous $1 \mathrm{~m}$ de chute

$$
Q_{1}=\left(\mathrm{Q} / \mathrm{H}_{n}\right)
$$

pour une valeur constante de :

où :

$$
n_{1}=\frac{\pi \mathrm{D}_{r} n}{60 \sqrt{2 g \mathrm{H}_{n}}}
$$

$\mathrm{D}_{r}=$ diamètre Pelton;

$n=$ vitesse de rotation;

$\mathrm{H}_{n}=$ chute nette.

$7^{\circ}$ Documents J. M. Vorth (G.m.b.H.).

Aucun effet d'échelle n'est constaté sur le rendement et sur le débit entre les chutes d'essais de 40 et $90 \mathrm{~m}$.

\section{B - Comparaison des résultats d'essais de modèles et de prototypes.}

Dans ce domaine, l'expérimentation nécessite la mise en œuvre de moyens considérables et il est très difficile d'entreprendre une campagne d'essais systématiques. La plupart des réponses émanent de construcleurs faisant état de résultats obtenus dans leurs laboratoires de recherches, d'une part, el de résultats d'essais de perception, d'autre part.

Dans ces conditions, le nombre de résultats dont il est possible de faire état est extrêmement faible. En effet, nous avions insisté dans le questionnaire sur la nécessité d'une parfaite similitude géométrique et nous pensons qu'il y a lieu de s'y tenir tant que des essais systématiques n'auront pas permis de définir quelles libertés il est possible de prendre. Or, les modèles de recherche sont le plus souvent réduits alux éléments essentiels afin d'être facilement et rapidement modifiables. Ils sont parfois constitués de pièces appartenant à un modèle précédent, de caractéristiques voisines. Pour toutes ces raisons, une similitude parfaite est rarement réalisée.

De plus, les conditions d'alimentation des modèles reproduisent rarement les conditions naturelles.

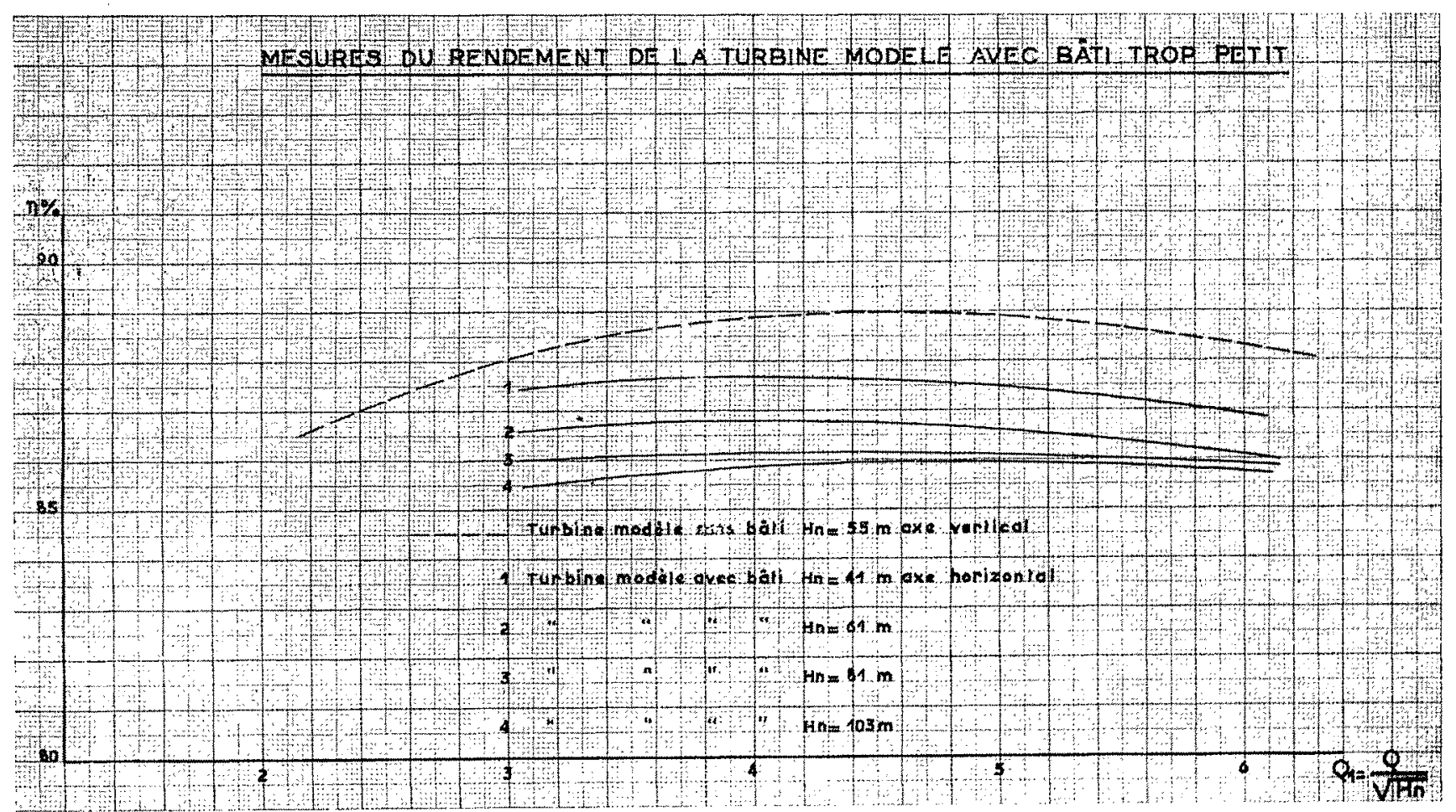

FiG. 3. 


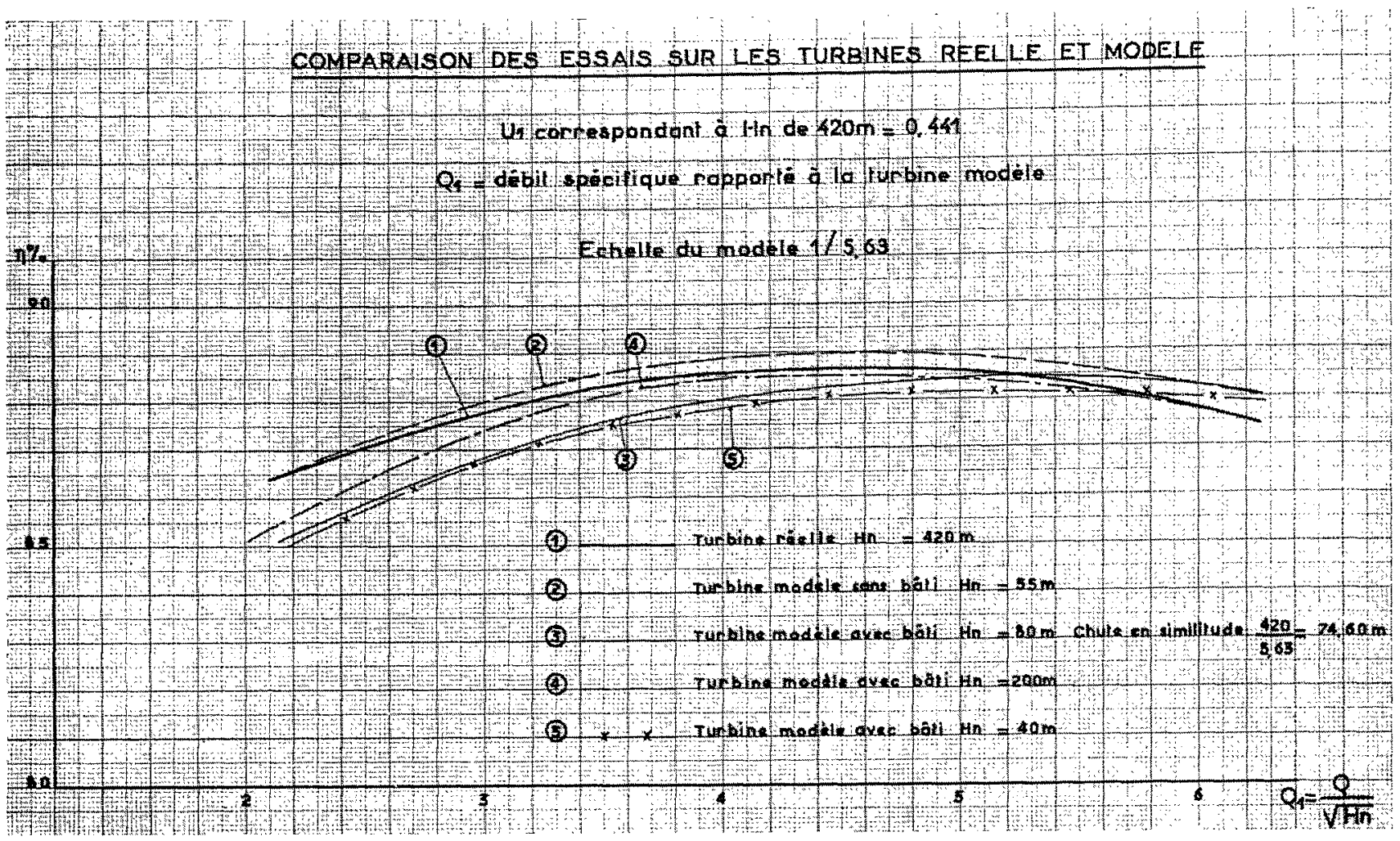

FrG. 4.

Les essais industriels mis en regard de ces essais sur modèle sont le plus souvent limités aux essais de réception, et sont rarement répétés, à cause des sujétions qu'ils imposent aux exploitants. Ces essais sont en général exécutés par le maître de l'œeuvre ou par un organisme indépendant et il semble qu'une grande diserétion soit de mise sur les résultats, alors que l'établissement de données certaines sur l'effet d'échelle ne peut résulter que de la confrontation d'un très grand nombre de cas, par suite de l'imprécision des mesures, surtout sur les machines industrielles.

En règle générale, les expérimentateurs ne respectent que la similitude des triangles de vitesse sans conditions supplémentaires. Certains auteurs font cependant remarquer l'importance de la similitude de Froude pour la représentation correcte des trajectoires de l'eau à la sortie de la roue.

\section{RESULTATS}

Nous pensons pouvoir faire état des résultats suivants.

$1^{\circ}$ Documents Charmiles (tirés de Informations techniques Charmilles (véf. $\mathrm{n}^{\circ} 8$ )).

Ces résultats sont relatifs aux essais d'une turbine à axe vertical à 4 jets dont les caractéristiques principales sont les suivantes :

\begin{tabular}{l|c|c} 
Diamètre Pelton........ (m) & 0,539 & 2,350 \\
Vitesse de rotation...(tr $/ \mathrm{mn})$ & 700 & 450 \\
Chute d'essai moyenne..(m) & 90 & 709 \\
Nombre d'injecteurs en service & 4 & 4
\end{tabular}

Le mode de présentation des résultats concernant les rendements est le même qu'au paragraphe II A 3 (voir fig. 5).

L'effet d'échelle sur les débits est mis en évidence par la figure 6 où le coefficient de débit $\mu$ est représenté en fonction de la course relative du pointeau.

$\mu$ est défini par $\mathrm{Q}=\mu \frac{\pi}{4} d_{e}^{2} \sqrt{2 g \mathrm{H}_{n}}$ où :

$d_{e}=$ diamètre d'embouchure;

$\mathrm{H}_{n}=$ chute nette.

La course du pointeau $\mathrm{C}_{p}$ est rapportée au diamètre d'embouchure de l'injecteur $d_{e}$.

\section{Remarque}

Pour les essais ci-dessus, le débit du prototype n'est pas mesuré, il est obtenu à partir des valeurs de $\mu$ communes à tous les injecteurs industriels. 
FiG. 5 .

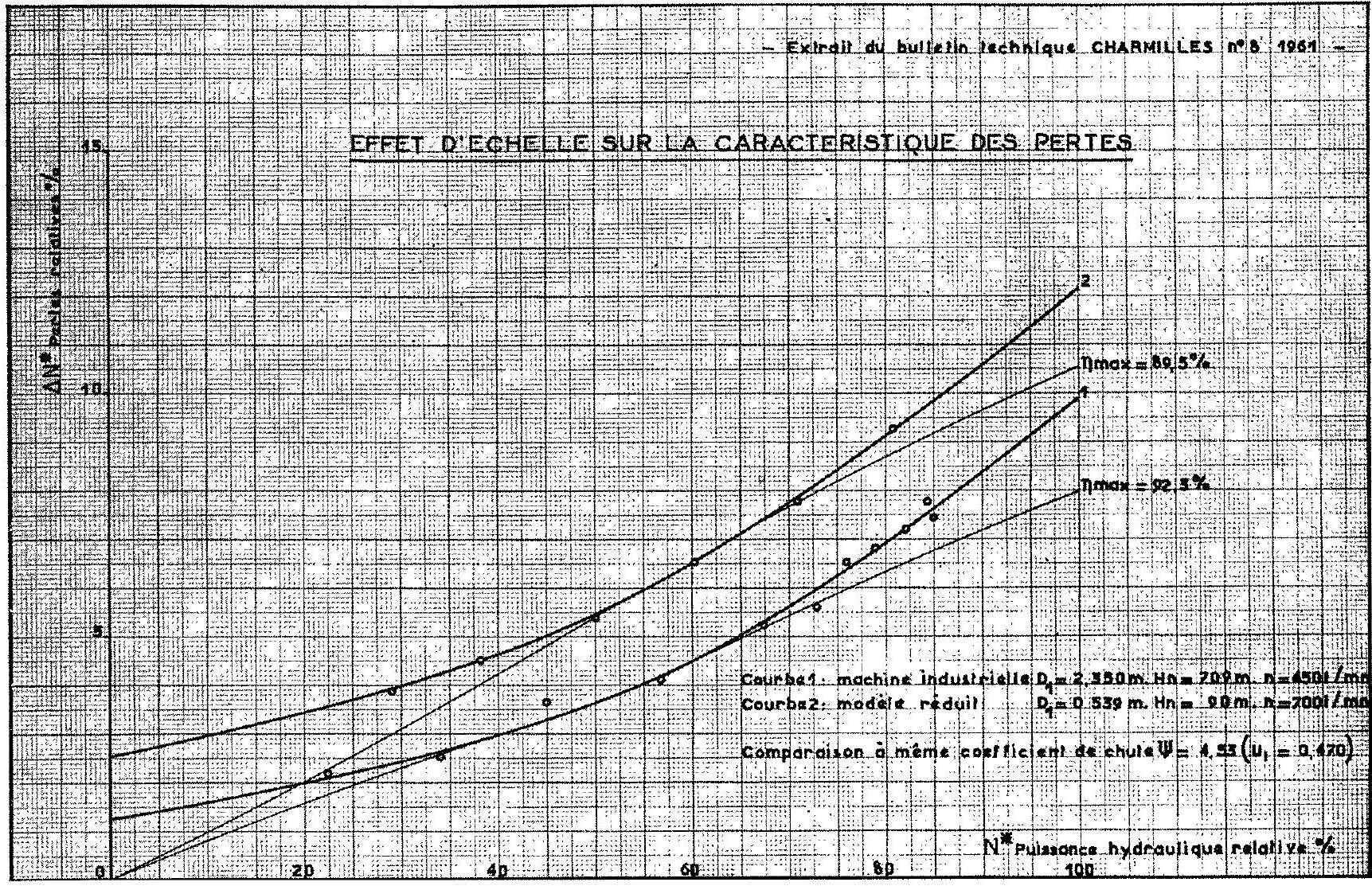

\section{COMPLÉMENTS}

Comparaison du coefficient de débit $\mu$ d'injecteurs industriels et d'injecteurs modèles.

Les cinq injecteurs industriels étudiés ont des diamètres d'embouchure variant de 120 à $300 \mathrm{~mm}$ et fonctionnent sous des chutes allant de 295 à $1700 \mathrm{~m}$. Pour tous ces injecteurs, il est possible de tracer une courbe unique donnant $\mu$ en fonction de $\mathrm{C}_{p} / d_{e}$.

L'injecteur modèle, de diamètre d'embouchure $d_{e}=51,4 \mathrm{~mm}$, est essayé sous 80 à $90 \mathrm{~m}$ de chute. Des valeurs de $\mu$ différentes sont obtenues suivant que l'injecteur est essayé seul sur une conduite rectiligne ou monté sur une volute à quatre injecteurs.

Le tableau suivant résume les résultats :

$\Delta \mu=\mu_{\text {modele }}-\mu_{\text {prototype }}$

Course relative...... $\quad C_{p} / d_{e}=0,6 \quad \mid \mathrm{C}_{p} / d_{e}=0,3$

Modèle :

- à quatre jets... $\frac{\Delta \mu}{\mu}=+0,87 \%+0,57 \%$

\begin{tabular}{l|l|l}
$-\dot{a}$ un jet. . . . & $+1,66 \%$ & $+1,29 \%$
\end{tabular}

Fitg. 6.

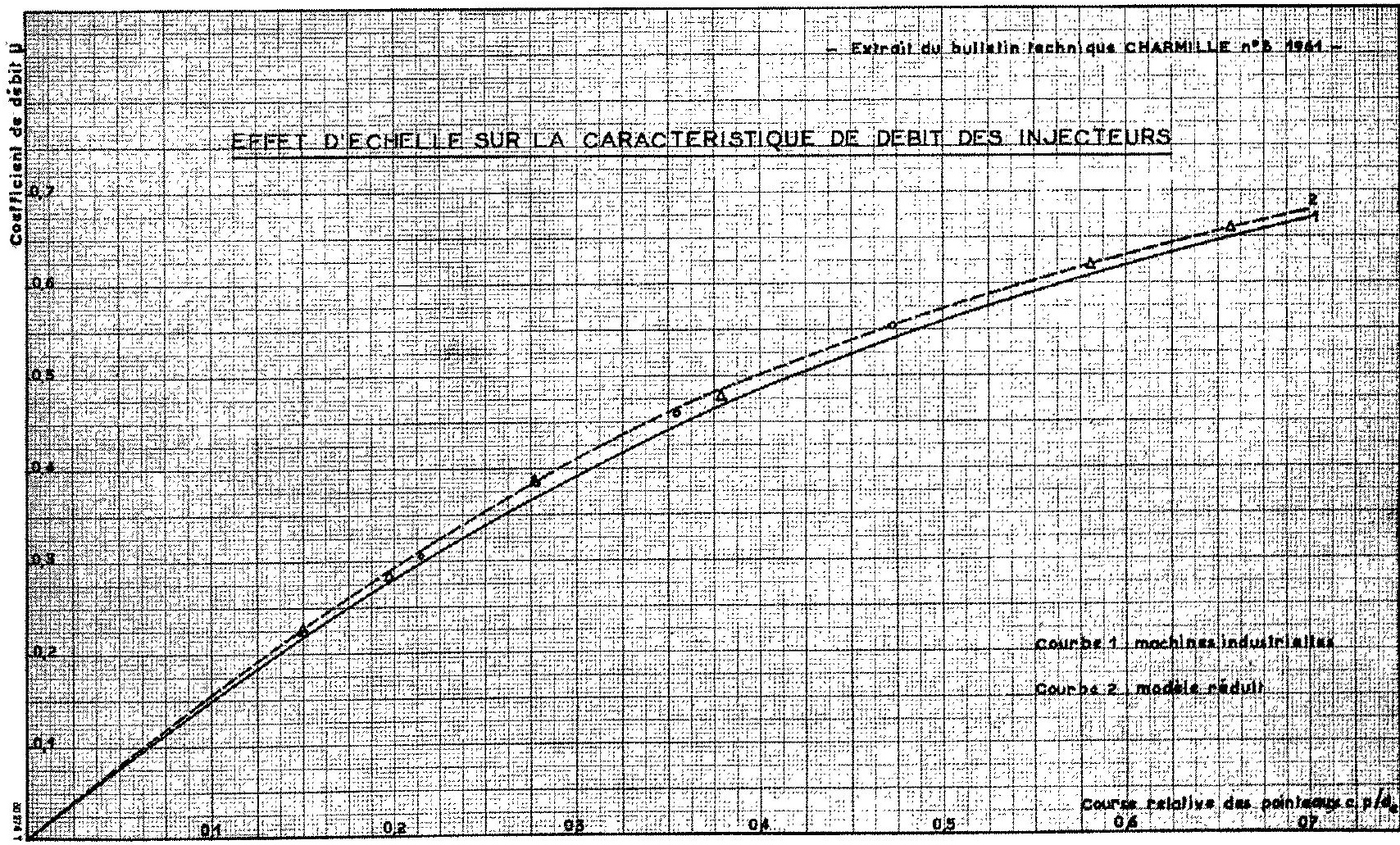


$2^{\circ}$ Documents Peiton (Division of Baldwin Lima Hamilton Corporation).

Turbine $\dot{a}$ axe horizontal à un injecteur

\begin{tabular}{|c|c|c|}
\hline & MODÈLE & PROTOTYPE \\
\hline Chute d'essai..... & 36,576 & 243,84 \\
\hline Diamètre Pelton........ (nm) & 546,1 & 1638 \\
\hline Diamètre de l'injecteur (mm) & 203,2 & 609,6 \\
\hline Rendement maximal..... (\% & 87,5 & 88 \\
\hline
\end{tabular}

\section{$3^{\circ}$ Documents SO.GR.E.A.H.}

Les résultats sont résumés dans les tableaux suivants :

Turbine à axe horizontal à deux jets

\begin{tabular}{|c|c|c|}
\hline & Modìle & PROTOTYPE \\
\hline 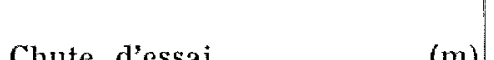 & & $-\frac{1}{488}$ \\
\hline ètre Pelton....... (m & 340 & 1175 \\
\hline Diamètre de l'injecteur (mm) & 140 & 450 \\
\hline
\end{tabular}

Défauts de similitude entre modèle et prototype :

Amenée: pas de culotte de branchement sur le modèle; amenée dans le plan de la roue, sur le modèle, oblique sur le prototype.

Augets légèrement plus épais sur le prototype. Augets rapportés sur le modèle seulement.

Fosse: pas de raccord entre le bâti et la fosse sur le modèle.

Injecteur : diamètre 140 au lieu de $130 \mathrm{~mm}$.

Ecarts : rendement prototype - rendement modèle :

\begin{tabular}{|c|c|}
\hline ChaRge & $\begin{array}{c}\text { ECART EN PoINTs } \\
\text { DE RENDEMENT }\end{array}$ \\
\hline $4 ! 10$ & $-0,6$ \\
$5 / 10$ & $-0,3$ \\
$6 / 10$ & $-0,7$ \\
$7 / 10$ & -1 \\
$8 / 10$ & $-1,5$ \\
$9 / 10$ & -2.2 \\
$10 / 10$ & -3 \\
\hline
\end{tabular}

Turbine à axe vertical à deux jets.

\section{Dimensions principales}

\begin{tabular}{|c|c|c|}
\hline & Modéle & PROTOTYPE \\
\hline Chute. . . . . . . . . . (m) & 60 & 1140 \\
\hline Diamètre Pelton...... (mm) & 497 & 3250 \\
\hline Diamètre d'injecteur.... (mm) & 105 & 710 \\
\hline
\end{tabular}

Défauts de similitude :

- la jante de la roue n'est pas en similitude, les augets sont rapportés sur le modèle seulement;
-_. le diamètre d'injecteur est $105 \mathrm{~mm}$ au lieu de $108,5 \mathrm{~mm}$.

-.. écarts : rendement prototype - rendement modèle :

\begin{tabular}{|c|c|}
\hline ChaRge & $\begin{array}{c}\text { EcART EN PoINTs } \\
\text { DE RENDEMEN }\end{array}$ \\
\hline $5 / 10$ & $+1,1$ \\
$6 / 10$ & $+0,5$ \\
$7 / 10$ & $+0,2$ \\
$8 / 10$ & 0 \\
$9 / 10$ & $-0,6$ \\
$10 / 10$ & $-0,8$ \\
\hline
\end{tabular}

Essais complémentaires :

In essai comparatif d'une roue à augets rapportés et de la même roue modifiée pour figurer une roue monobloc n'a montré aucun écart de. rendement.

Effet d'échelle sur les débits :

Caractéristiques des injecteurs essayés

\begin{tabular}{|c|c|c|c|}
\hline & MOJİLE & PROT & TYPE \\
\hline & - & - & \\
\hline Chute. . . . . . . . . (m) & 60 & 475 & 915 \\
\hline Diamètre de l'injecteur (mm) & 108,5 & 450 & 755 \\
\hline
\end{tabular}

Il résulte de cette comparaison que les coefficients de débit des injecteurs industriels sont toujours inférieurs à ceux des modèles, l'écart se situant entre 0 et $5 \%$. La dispersion des mesures est assez grande.

\section{$4^{\circ}$ Documents Vevey.}

Sur la figure 4, figure également le rendement du prototype semblable au modèle décrit au paragraphe II A 6 .

L'échelle du mođèle est $1 / 5,63$.

Les notations sont expliquées au paragraphe II A 6 .

\section{$5^{\circ}$ Documents J. M. Voith.}

a) Turbine à axe vertical (fig. 7).

Dimensions du prototype :

- chute : $900 \mathrm{~m}$;

- nombre d'injecteurs : 4;

- largeur d'auget : $0,388 \mathrm{~m}$;

-.- diamètre Pelton : $1,630 \mathrm{~m}$;

- échelle du modèle : $1 / 3,43$;

- échelle de la chute : 1/11,2.

Le graphique représente le rendement rapporté au rendement maximal du modèle, en fonction du débit rapporté au débit de pleine char'ge. 
Fig. 7.

\section{TURGINE N AXE VERTICAL}

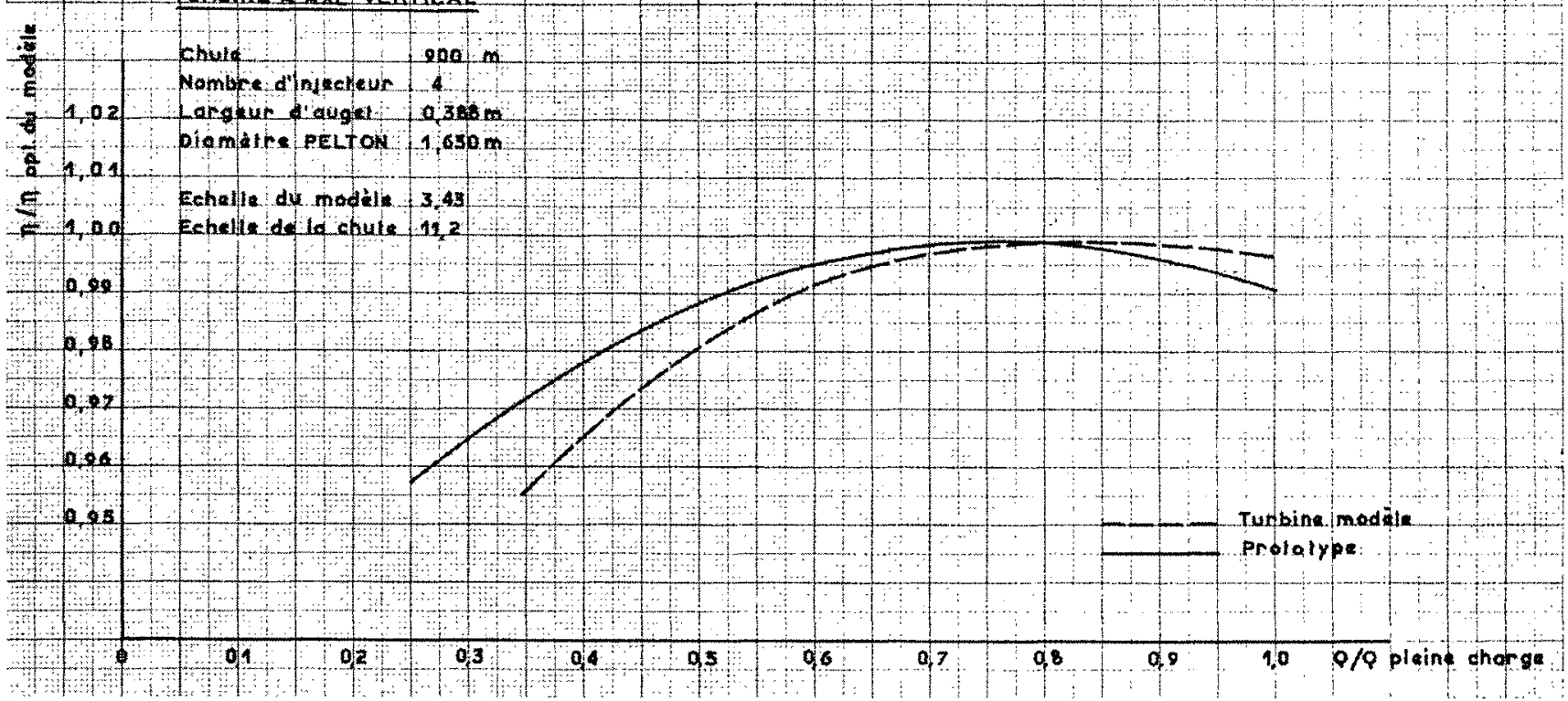

b) Turbine à axe horizontal à 2 jets (fig. 8).

Dimensions du prototype :

- hauteur de chute : $940 \mathrm{~m}$;

-- nombre d'injecteurs : 2;

- largeur d'auget : $0,510 \mathrm{~m}$;

- diamètre Pelton : 2,540 m;

- échelle du modèle : 1/3,44;

- échelle de la chute : 1/11,7.

c) 'Turbine à axe vertical à 6 jels (fig. 9 ).

Dimensions du prototype :

- hauteur de chute : $540 \mathrm{~m}$; rendement optimum du modele $=1$

$-1+y^{-}-1$


Fic. 12. Comparaison modèle-prototype.

conduit aux écarts suivants sur le coefficient de débit :

\begin{tabular}{l|c|c|c|c} 
- orverture. . & $1 / 4$ & $1 / 2$ & $3 / 4$ & 1
\end{tabular}

\begin{tabular}{ll|l|l|l} 
- écart. . . . . & 0 & 1 & 2 & $2,15 \%$
\end{tabular}

Le coefficient de débit est plus faible sur l'injecteur de grandes dimensions.

\section{III. - INTERPRETATION DES RE- SULTATS.}

\section{Séparation des pertes}

Il résulte du paragraphe précédent que, si des écarts de rendement sont
TURBINE A AXE NERTICAL

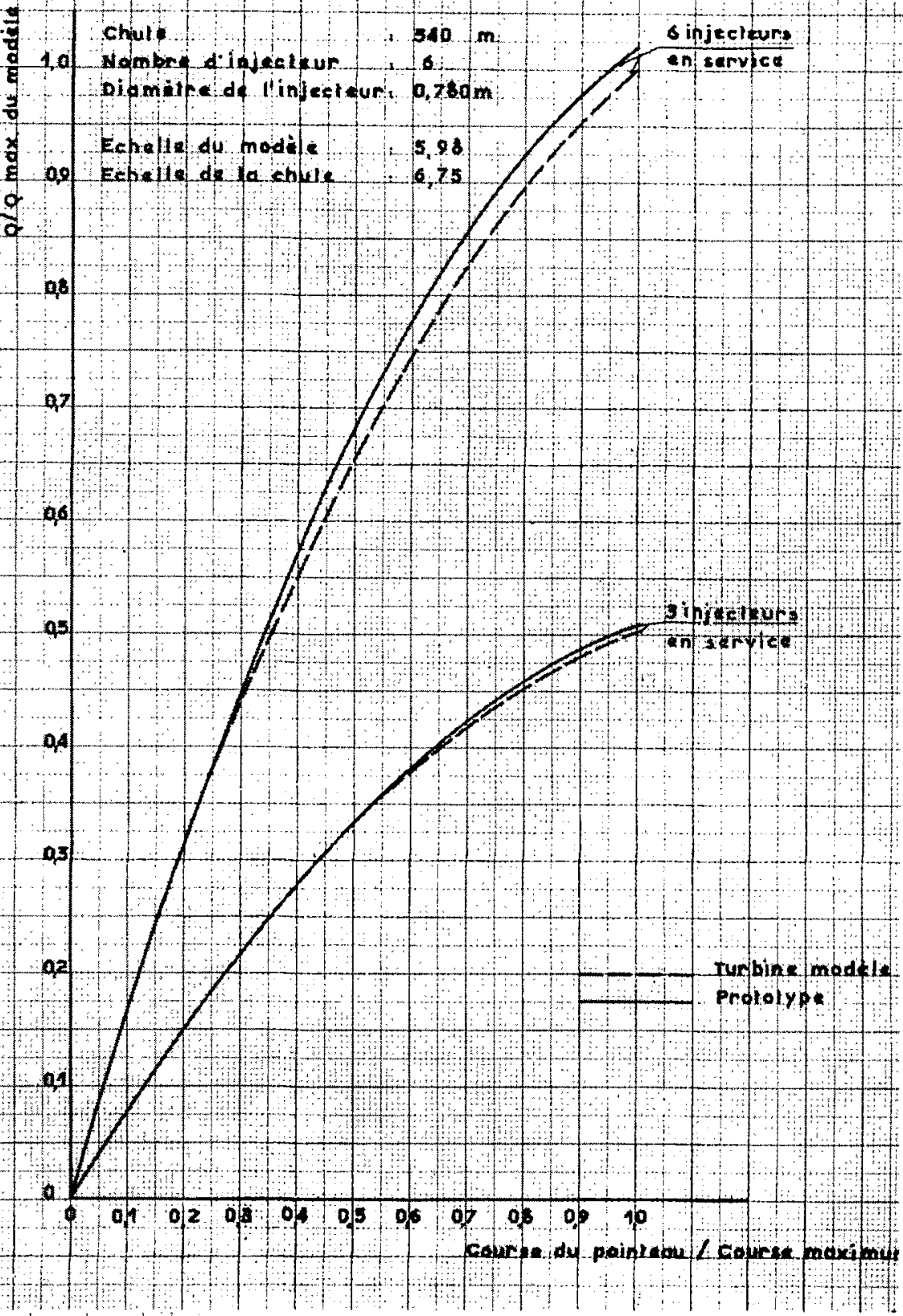

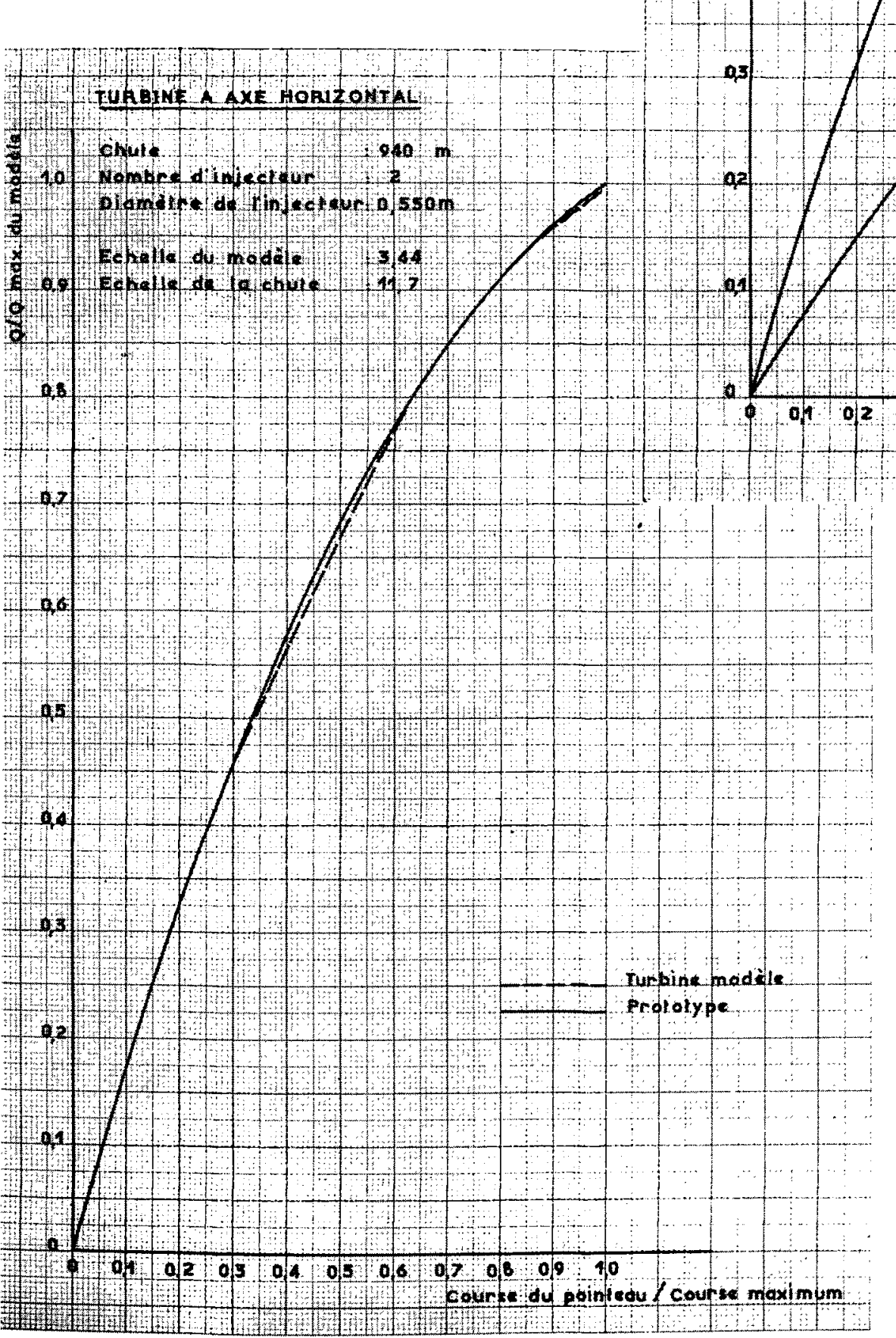

fréquemment constatés, il ne se dégage aucune évidence quant à la cause de ces écarts, qui sont parfois de sens opposés.

Certains essais mettent en évidence un effet d'échelle positif sur le rendement quand on passe du modèle au prototype, comme dans les turbines à réaction; d'autres montrent un effet négatif.

Dans ce dernier cas, les essais réalisés a Vevey (fig. 3) semblent montrer que nous sommes en présence d'un effet perturbateur dû à un bâti-capote mal dimensionné. 
Dans un tel cas, il est évidemment nécessaire de s'en tenir à la similitude de Froude si l'on veut reproduire correctement les trajectoires de l'eau à la sortie de la roue. Cette condition n'est évidemment pas suffisante pour obtenir le méme rendement sur modèle et sur protoiype, puisque les pertes engendrées par le bâti-capote peuvent néanmoins conserver une importance relative différ ente dans les deux cas.

Si le bâti-capote est dimensionné de facon à ne jouer aucun rôle perturbateur dans le fonctionnement de la roue, il est probable que la réalisation de la similitude de Froude, n'est plus nécessaire, du moins dans une certaine gamme de chutes d'essais, et que l'on doit se retrouver en présence d'un effet d'échelle posilif, mais beaucoup plus faible, dù à la variation des pertes par frottement. Cette hypothèse semble confirmée par les remarques suivantes:

- les turbines à axe vertical pour lesquelles l'évacuation de l'eau est meilleure et les risques de rejaillissement sont plus faibles présentent pratiquement toutes un effet d'échelle positif;

- pour les turbines à effet d'échelle positif, celui-ci est plus fort aux faibles charges, où il est vraisemblable que les pertes par frottement présentent une importance relative plus grande,

Il serait évidemment souhaitable d'effectuer une analyse plus poussée des différentes pertes et peu d'essais ont été réalisés dans ce sens.

Mis à part les frottements dans l'air, qu'il est facile de mesurer et qui sont du reste relativement faibles, les pertes restent difficiles à séparer.

Il faut citer à ce sujet les travaux effectués par E.D.F. [réf. $n^{\circ} 9$ ]. La méthode thermodynamique de mesure de rendement permet, en prélevant l'eau directement à la sortie de la rouc, de mesurer un rendement fonclionnel, injecteurauget. Les pertes «supplémentaires» mises en évidence peuvent ètre attribućes à l'énergie cinétique de l'eau pulvérisée, qui quitte la roue avec une vitesse absolue voisine de la vitesse périphérique de celle-ci et travaille dans la turbine avec un rendement voisin de $50 \%$.

Des essais ont été entrepris pour déterminer la quantilé d'eau pulvérisée en suspension sous le bâti-capote d'une turbine par mesure de l'absorption du rayonnement $\beta$. Après une mise au point en laboratoire, rendue nécessaire par les difficultés rencontrées dans l'emploi de cette méthode, des essais pourraient être entrepris aussi bien sur modèle réduit que sur turbine industrielle,

\section{IV. - AUTRES ETUDES THÉORIQUES ET EXPERIMENTALES}

Parmi les études théoriques ou expérimentales pouvant être rattachées au problème de l'eflet d'échelle dans les turbines Pelton, nous ne pouvons citer que les travaux entrepris au Laboratoire de Mécanique des fluides de l'Université de Grenoble [réf. $n^{\circ} 1$ ].

Cette étude porte sur la mesure de la dispersion d'un jet et sur les variations de cette dispersion en fonction des nombres de Reynolds et de Weber.

\section{CONCLUSIONS}

En ce qui concerne les débits, les auteurs sont en général d'accord, sinon sur la valeur, du moins sur le sens de l'effet d'échelle avec les résultats publiés par $H$. Gerber [réf. $n^{\circ} 5$ ].

Par contre, à la fin de cet exposé, il faut bien reconnaitre qu'une assez grande confusion règne en ce qui concerne les rendements, et c'est pourtant là que se trouve le problème le plus important.

Nous ne pensons pas que la réunion d'un bien plus grand nombre de documents analogues à ceux présentés ici soit possible, ni qu'elle permette une étude statistique de l'effet d'échelle car, en général, trop de doutes subsistent quant à la précision des mesures ou à la similitude des machines comparées.

Une étude systématique plus fine des pertes dans un certain nombre de couples modèle. prototype serait certainement plus efficace.

Le problème de l'effet d'échelle dans les turbines Pelton existe donc réellement, mais il semble qu'il se présente sous une forme encore plus complexe que dans les turbines à réaction. Pour ces dernières, les formules de transposition ne tiennent en général compte que des pertes cinétiques et des pertes par frottement et donnent des résultats qui coincident assez bien avec l'expérience.

Ici, les mécanismes mis en jeu sont beaucoup plus complexes du fait de l'écoulement à l'air libre; par exemple, la dispersion du jet, la pulvérisation de l'eau, les rebondissements d'eau sur le bâti-capote, les frottements de Ia roue dans l'air chargé d'eau en suspension peuvent intervenir dans des proportions variables et i] est impossible d'envisager une formule de transposition tenant compte de tels paramètres dans l'état actuel des études.

Le professeur Gerber cite [réf. $n^{\circ} 6$ ] l'application heureuse de la formule de Grégorig à une 
turbine à action à jet annulaire. Il est permis de penser que, suivant l'hypothèse avancée au paragraphe III, nous nous trouvons dans un cas où l'effet d'échelle positif dépendant du nombre de Reynolds se manifeste de façon prépondérante. C'est peut-être en se limitant à ce seul cas qu'il convient pour l'instant de chercher à établir une formule de transposition.

En souhaitant recevoir de nombreux éléments susceptibles d'être ajoutés à ce mémoire, nous remercions encore ici toutes les personnes qui, déjà, ont bien voulu y participer.

\title{
DISCUSSION
}

Président : M. Bergeron

M. le Président remercie M. Vercasson de l'effort qu'il a fait et attire l'attention sur l'importance du rapport qu'il a établi.

Cette communication indique la prise de position de la Section «Machines» sur l'impossibilité actuelle de donner une conclusion précise concernant l'effet d'échelle dans les essais sur modèle des turbines Pelton.

M. le Président aurait aimé que quelques personnes de l'assemblèe donnent leur avis sur cette conclusion, qui peut avoir aussi une importance dans les rapports entre le constructeur et l'utilisateur.

\section{Collated data on the scale effect on Pelton turbine efficiency and discharge}

\author{
BY M. VERCASSON,
}

\author{
ABSTRACT
}

\section{INTRODUCTION}

This investigation was undertaken at the request of the Hydraulic Machines Committee of the Société Hydrotechnique de France.

Far less scale effect research has been done on Pelton turbines than on reaction turbines and this research has in any case never produced any practical transposition formula.

This being so, it may well be asked if a problem does in fact exist, especially in view of the following remark in the International Electrotechnical Commission's Code of Test Procedure, paragraph 3.222, for acceptance tests on scale models:

"No scale effect formula for Pelton turbines: prototype efficiency = model efficiency."

The collected references in fact show that this state of affairs is due to the divergence of scale effect results observed by various experimenters, which justifies the present study.

The work of collating all this various information necessitated sending a questionnaire to twenty-four individuals or groups throughout Western Europe and North America, who are interested in this problem.

The questionnaire was divided into four paragraphs, as follows:

\section{I.-REFERENCES (see French text)}

\section{II.-TEST RESULTS SHOWING SCALE EFFECT ON BOTH DISCHARGES AND EFFICIENCY}

This section was divided into two, to show, on the one hand the effect of variations in head taken in isolation, and, on the other, the effect of simultaneously varying both head and turbine dimensions. 


\section{A - Tests on one turbine under different heads.}

This type of test is obviously the easiest to carry out accurately, at least on a scale model. Figures $1,2,3$, and 4 give examples of results obtained using the same model under different heads.

\section{B - Comparison of test results on model and prototypes.}

Experiments in this field require the use of extensive test equipment and it is extremely difficult to carry out a systematic series of measurements. The majority of the replies supplied by manufacturers concerned either the results obtained in their own research laboratories or during acceptance tests.

This paragraph compares model and prototype efficiencies based on replies from five different laboratories. (Cf. especially figures $4,5,7,8$, and 9 ).

Figures $6,10,11$ and 12 allow a comparison of model and prototype nozzle discharge coefficients to be made.

As a general rule the experimenters only observe velocity triangle similitude, although some do point out the importance of Froude similitude if the trajectory of the water on leaving the runner is to be correctly reproduced.

\section{III-INTERPRETATION OF THE RESULTS: ANALYSIS OF EFFICIENCY LOSSES}

The previous section offers no obvious interpretation of the deviations observed in efficiency figures, which are often contradictory.

In one case only was it possible to give practically conclusive proof of the disturbing effect on runner operation of a badly-dimensioned housing. In this case Froude similitude would obviously have to be observed to ensure correct reproduction of the exit trajectories.

If the dimensions of the housing are such that disturbing effects are eliminated Froude similitude may probably be dispensed with, at least for certain range of test heads and a positive scale effect should be observed. This scale effect will however be much less marked owing to variations in friction loss. This hypothesis would seem to be borne out by the following remarks:

$1^{\circ}$ Vertical shaft turbines where the evacuation is better and risk of splash less, almost all show a positive scale effect.

$2^{\circ}$ In the case of turbines with positive scale effect the latter is more marked under low heads, probably because friction losses are relatively more important.

No complete method of efficiency Ioss analysis is given, except in the case of friction losses due to air, which are easily measurable and in any case relatively slight.

\section{IV,-OTHER THEORETICAL AND EXPERIMENTAL STUDIES}

Among the theoretical and experimental studies which have a bearing on the problem of scale effect in Pelton turbines mention should be made of the work carried out at the Fluid Mechanics Laboratories of Grenoble University. This study concerns the measurement of the dispersion of a jet and its variations in terms of Reynolds and Weber numbers.

\section{CONCLUSIONS}

As fare as discharges are concerned the authors are generally agreed if not as to the value, at least as to the trend of the scale effect. As far as efficiency is concerned, on the other hand, the end of this paper reveals a good measure of confusion, despite the fact that this is the really important problem.

It would not be casy to assemble a larger collection of similar documents nor would this enable a statistical study to be done, since there is generally speaking too much doubt as to the accuracy of the results or the similitude of the machines compared.

A closer systematic study of losses in a certain number of model-prototype pairs would certainly be more effective. 